
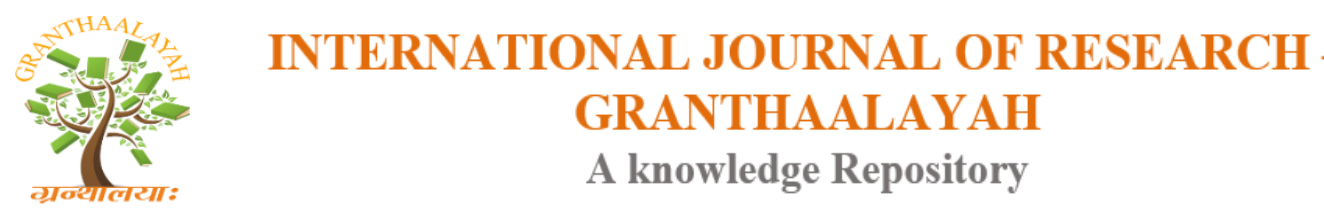

Science

\title{
ENHANCED SPIDER ALGORITHM FOR MINIMIZATION OF REAL POWER LOSS
}

\author{
Dr. K. Lenin *1 \\ ${ }^{* 1}$ Professor, Department of EEE, Prasad V.Potluri Siddhartha Institute of Technology, Kanuru, \\ Vijayawada, Andhra Pradesh -520007, India
}

\begin{abstract}
In this paper Enhanced Spider (ES) algorithm is proposed to solve reactive power Problem. Enthused by the spiders, a new Enhanced Spider (ES) algorithm is utilized to solve reactive power problem. The composition is primarily based on the foraging approach of social spiders, which make use of of the vibrations spread over the spider web to choose the position of prey. The simulation results demonstrate high-quality performance of Enhanced Spider (ES) algorithm in solving reactive power problem. The projected Enhanced Spider (ES) algorithm has been tested in standard IEEE 57,118 bus systems and compared to other reported standard algorithms. Results show that Enhanced Spider (ES) algorithm is more efficient than other algorithms in reducing the real power loss.
\end{abstract}

Keywords: Enhanced Spider Algorithm; Swarm Intelligence; Reactive Power; Transmission Loss.

Cite This Article: Dr. K. Lenin. (2018). "ENHANCED SPIDER ALGORITHM FOR MINIMIZATION OF REAL POWER LOSS." International Journal of Research - Granthaalayah, 6(4), 301-311. https://doi.org/10.29121/granthaalayah.v6.i4.2018.1665.

\section{Introduction}

In recent years the reactive power problem has received huge attention as a result of the enhancement on economy and security of power system operation. Solutions of reactive power problem intend to minimize object functions such as fuel cost, power system loses, etc. while satisfying a number of constraints like limits of bus voltages, tap settings of transformers, reactive and active power of power resources and transmission lines and a number of controllable Variables $[1,2]$. In the literature, many methods for solving the ORPD problem have been done up to now. At the beginning, several classical methods such as gradient based [3], interior point [4], linear programming [5] and quadratic programming [6] have been effectively used in order to solve the ORPD problem. However, these methods have some disadvantages in the procedure of solving the complex ORPD problem. Drawbacks of these algorithms can be declared insecure convergence properties, extended execution time, and algorithmic intricacy. In addition, the solution can be trapped in local minima $[1,7]$. In order to triumph over these disadvantages, researches have been effectively applied evolutionary and heuristic algorithms such as Genetic Algorithm (GA) [2], 
Differential Evolution (DE) [8] and Particle Swarm Optimization (PSO) [9]. Voltage stability evaluation using modal analysis [10] is used as the indicator of voltage stability. Spiders is airbreathing arthropods. They have eight legs and chelicerae with fangs. They use an extensive range of different strategies for foraging, and most of them sense prey by sensing vibrations. Spiders have long been known to be very responsive to vibratory stimulation, as vibrations on their webs notify them of the capture of prey. If the vibrations are in a defined range of frequency, spiders attack the vibration source. The social spiders can also distinguish vibrations generated by the prey with ones generated by other spiders [11]. The performance Enhanced Spider (ES) algorithm of has been evaluated in standard IEEE 57,118 bus systems and the results analysis shows that our proposed approach outperforms all approaches investigated in this paper.

\section{Objective Function}

\section{Active power loss}

Main aim of the reactive power dispatch problem is to reduce the active power loss in the transmission network, which can be described as:

$\mathrm{F}=\mathrm{PL}=\sum_{\mathrm{k} \in \mathrm{Nbr}} \mathrm{g}_{\mathrm{k}}\left(\mathrm{V}_{\mathrm{i}}^{2}+\mathrm{V}_{\mathrm{j}}^{2}-2 \mathrm{~V}_{\mathrm{i}} \mathrm{V}_{\mathrm{j}} \cos \theta_{\mathrm{ij}}\right)$

Where gk: is the conductance of branch between nodes i and j, Nbr: is the total number of transmission lines in power systems.

\section{Voltage profile improvement}

For minimization of the voltage deviation in PQ buses, the objective function turns into:

$\mathrm{F}=\mathrm{PL}+\omega_{\mathrm{v}} \times \mathrm{VD}$

Where $\omega \mathrm{v}$ : is a weighting factor of voltage deviation.

$\mathrm{VD}$ is the voltage deviation given by:

$\mathrm{VD}=\sum_{\mathrm{i}=1}^{\mathrm{Npq}}\left|\mathrm{V}_{\mathrm{i}}-1\right|$

\section{Equality Constraint}

The equality constraint of the Reactive power problem is represented by the power balance equation, and can be written as, where the total power generation must cover the total power demand and total power loss:

$P_{G}=P_{D}+P_{L}$

Where, $\mathrm{P}_{\mathrm{G}}$ - Total Power Generation, $\mathrm{P}_{\mathrm{D}}$-Total Power Demand, $\mathrm{P}_{\mathrm{L}}-$ Total Power Loss.

\section{Inequality Constraints}


Inequality constraints define the limitations in power system components and power system security. Upper and lower bounds on the active power of slack bus, and reactive power of generators are written as follows:

$\mathrm{P}_{\text {gslack }}^{\min } \leq \mathrm{P}_{\text {gslack }} \leq \mathrm{P}_{\text {gslack }}^{\max }$

$\mathrm{Q}_{\mathrm{gi}}^{\min } \leq \mathrm{Q}_{\mathrm{gi}} \leq \mathrm{Q}_{\mathrm{gi}}^{\max }, \mathrm{i} \in \mathrm{N}_{\mathrm{g}}$

Upper and lower bounds on the bus voltage magnitudes are described as follows:

$V_{i}^{\min } \leq V_{i} \leq V_{i}^{\max }, i \in N$

Upper and lower bounds on the transformers tap ratios are given as follows:

$\mathrm{T}_{\mathrm{i}}^{\min } \leq \mathrm{T}_{\mathrm{i}} \leq \mathrm{T}_{\mathrm{i}}^{\max }, \mathrm{i} \in \mathrm{N}_{\mathrm{T}}$

Upper and lower bounds on the compensators reactive powers are written as follows:

$\mathrm{Q}_{\mathrm{C}}^{\min } \leq \mathrm{Q}_{\mathrm{c}} \leq \mathrm{Q}_{\mathrm{C}}^{\max }, \mathrm{i} \in \mathrm{N}_{\mathrm{C}}$

Where $\mathrm{N}$ is the total number of buses, NT is the total number of Transformers; Nc is the total number of shunt reactive compensators.

\section{Enhanced Spider Algorithm}

The spiders can move liberally on the web. However, they cannot go away from the web as the positions off the web represent infeasible solutions to the optimization problem. When a spider shifts to a new position, it creates a vibration which is propagated over the web. Each vibration holds the information of one spider and other spiders can get the information upon receiving the vibration.

\subsection{Spider}

At the beginning of the Enhanced Spider (ES) algorithm, a pre-defined number of spiders are placed on the web. Each spider holds a memory, storing the following individual information:

1) The location of spiders on the web.

2) The fitness of the present position of spiders.

3) The goal vibration of spiders in the previous iteration.

The first two types of information explain the individual situation of spiders, while the third type of information is concerned in directing spiders to new-fangled positions.

Based on observations, spiders are found to have very precise senses of vibration. In addition, they can divide different vibrations promulgated on the same web and sense their relevant intensities. Spider will create a vibration when it reaches a new-fangled position different from the previous one. The concentration of the vibration is connected with the fitness of the position. The vibration 
will propagate over the web and other spiders can sense it. In such a way, the spiders on the same web distribute their personal information with others to form a combined social knowledge.

\subsection{Vibration}

Vibration is a very noteworthy concept in Enhanced Spider (ES) algorithm. It is one of the key characteristics that distinguish Enhanced Spider (ES) algorithm from other algorithms. In Enhanced Spider (ES) algorithm, we use two properties to describe a vibration, namely the source position and the source concentration of the vibration. The source position is defined by the explore space of the optimization problem, and we define the concentration of a vibration in the range [0, $+\infty$ ]. Every time a spider moves to a new position, it produces a vibration at its present position. We define the position of spider a at time $\mathrm{t}$ as $\mathrm{Pa}(\mathrm{t})$, or simply as $\mathrm{Pa}$ if the argument is $\mathrm{t}$. We further use $\mathrm{I}(\mathrm{Pa}, \mathrm{Pb}, \mathrm{t})$ to represent the vibration concentration sensed by a spider at position $\mathrm{Pb}$ at time $\mathrm{t}$ and the source of the vibration is at position Pa. Thus I (Ps, Ps, $t$ ) defines the concentration of the vibration created by spider $s$ at the source position. This vibration concentration at the source position is associated with the fitness of this position $\mathrm{f}(\mathrm{Ps})$, and we define the concentration value as follows:

$I\left(P_{s}, P_{s}, t\right)=\left\{\begin{array}{l}1 /\left(C_{\max }-f\left(P_{s}\right)\right) \text { for maximization } \\ 1 /\left(f\left(P_{s}\right)-C_{\text {min }}\right) \text { for minimization }\end{array}\right.$

Where Cmax is a confidently large constant selected such that all possible fitness values of the maximization problem are smaller than Cmax, and Cmin is a assertively small constant such that all possible fitness values of the minimization problem is larger than Cmin. Equation (24) guarantees that the probable vibration intensities of any optimization problem are all positive values. It further assurance that a better fitness value, i.e. larger for maximization or smaller for minimization problem, corresponds to larger vibration concentration.

\subsection{Intensity Attenuation}

As a form of energy, vibration attenuates over time and distance. This physical occurrence is accounted for in the design of Enhanced Spider (ES) algorithm by two equations,

1) Attenuation over Distance: We define the vibration attenuation over distance as follows. We describe the distance between spider $\mathrm{a}$ and $\mathrm{b}$ as $\mathrm{D}(\mathrm{Pa}, \mathrm{Pb})$, and the maximum distance between two points in the search space as Dmax. The description of Dmax can be problem dependent, and we use the following equation for simplicity:

$\mathrm{D}_{\max }=\|\overline{\mathrm{x}}-\underline{\mathrm{x}}\|_{\mathrm{p}}$

Where $\overline{\mathrm{x}}$ is the upper bound of the search space and $\underline{\mathrm{x}}$ is the lower bound of the search space. $\mathrm{p}$ indicates that we use p-norm as the technique to compute the distance between spiders, i.e.,

$\mathrm{D}\left(\mathrm{P}_{\mathrm{a}}, \mathrm{P}_{\mathrm{b}}\right)=\left\|\mathrm{P}_{\mathrm{a}}-\mathrm{P}_{\mathrm{b}}\right\|_{\mathrm{p}}$ 
In this paper we use 1-norm or Manhattan norm in distance calculation. If the search space is not constrained, $\overline{\mathrm{x}}$ and $\underline{\mathrm{x}}$ in Eqn. (25) stand for the upper and lower bound of the initial solution generation space, respectively. With the above definitions, we define the vibration attenuation over distance as follows:

$I\left(P_{a}, P_{b}, t\right)=I\left(P_{a}, P_{a}, t\right) \times \exp \left(-\frac{D\left(P_{a}, P_{b}\right)}{D_{\max } \times r_{a}}\right)$

In the above formula we introduce a user-controlled parameter ra $\in(0,1)$. This parameter controls the attenuation rate of the vibration concentration over distance. The larger ra is, the weaker the attenuation forced on the vibration.

2) Attenuation over Time: We also bring in an equation to model vibration attenuation over time. As the vibration biases other spiders to move, a non-decaying vibration may potentially draw other spiders incessantly, causing the algorithm to converge pre-maturely. So the power of previous vibrations shall be properly attenuated to prevent pre-mature convergence. The vibration attenuation over time is defined as follows:

$I\left(P_{a}(t), P_{a}(t), t+1\right)=I\left(P_{a}, P_{a}, t\right) \times r_{a}$

In each iteration, all vibrations created in the previous iteration are attenuated by the factor ra. We utilize the same parameter ra introduced in the vibration attenuation over distance formula for ease of parameter tuning. At time $t+1$, the location of spider a may change to $\mathrm{Pa}(\mathrm{t}+1)$, but the source position of the vibration remains at $\mathrm{Pa}(\mathrm{t})$.

\subsection{Search Pattern}

Here we express the above thoughts in terms of an algorithm. There are three phases in Enhanced Spider (ES) algorithm: initialize, iteration, and conclusion. These three phases are executed successively. In every run of ES algorithm, we begin with the initialize stage, then execute searching in an iterative manner, and lastly stop the algorithm and output the solutions found. In the initialize stage, the algorithm describes the objective function and its solution space. As the number of spiders remains unchanged during the simulation of Enhanced Spider (ES) algorithm, a fixed size memory is allocated to accumulate their information. The location of spiders is arbitrarily created in the explore space, with their fitness values calculated and stored. The target vibration of each spider in the population is set at its current position, and the vibration concentration is zero. This ends the initialize stage and the algorithm starts the iteration stage, which execute the search with the artificial spiders produced. In the iteration phase, a number of iterations are executed by the algorithm. In each iteration, all spiders on the web shift to a new position and calculate their fitness values. The algorithm first computes the fitness values of all the artificial spiders on different positions on the web. Then these spiders generate vibrations at their locations using Equation (10). After all the vibrations are created, the algorithm simulates the propagation method of these vibrations using Equation (13). In this procedure, each spider s will accept pop Size -1 different vibrations created by other spiders. The received information of these vibrations includes the source position of the vibration and its attenuated concentration. We use $\mathrm{V}$ to symbolize these pop Size -1 vibrations. Upon the receipt of $\mathrm{V}$, s will select the strongest vibration vbest from $\mathrm{V}$ and compare its strength with the concentration of the target vibration vtar 
stored in its memory. s will store vbest as vtar if the intensity of vbest is larger, otherwise the original vtar is preserved.

$\mathrm{P}_{\mathrm{s}}(\mathrm{t}+1)=\mathrm{P}_{\mathrm{s}}+\left(\mathrm{P}_{\mathrm{tar}}-\mathrm{P}_{\mathrm{s}}\right) \odot(1-\mathrm{R} \odot \mathrm{R})$,

Where $\odot$ denotes element-wise multiplication. Ptar is the vibration source location of the target vibration vtar. $\mathrm{R}$ is a vector of arbitrary numbers generated from zero to one uniformly, whose length is weak, and 1 is a vector of one's of length weak. The algorithm repeats this process for all the spiders in pop. To avoid Enhanced Spider (ES) algorithm getting stuck in a local optimum, we initiate an artificial spider jump away process. Each spider in pop, right after the arbitrary walk step, has a small probability to decide not to follow its present target and jump away from its current position. The probability is defined using the following equation:

$P_{j}=\frac{r_{j}}{\exp \left(D\left(P_{s}, P_{t a r}\right) / D_{\max }\right)}$,

Where rj is a user-defined jump away rate parameter. If spider $\mathrm{s}$ is chosen to jump away, a new arbitrary position in the explore space is generated and assigned as the new position of $s$. The last step of the algorithm is to attenuate the concentration of the stored target vibration using Equation (13) and this end the iteration phase. The iteration phase loops until the end criteria are matched. The stop criteria can be defined as the maximum iteration number reached, the maximum CPU time used, the error rate reached, the maximum number of iterations with no development on the best fitness value. After the iteration phase, the algorithm outputs the best solution with the best fitness established.

The above three phases comprise the complete algorithm of Enhanced Spider (ES) algorithm.

The proposed Enhanced Spider (ES) algorithm has binary mask based arbitrary walk used to engender new solutions for the next iteration has been replaced with the following mutation process, after which a selection process is applied.

$\mathrm{p}_{\mathrm{i}}^{\text {trail }}=\mathrm{p}_{\mathrm{i}}^{\text {target }}+\mathrm{r}_{\mathrm{i}} \times \mathrm{k}_{\mathrm{i}} \otimes\left(\mathrm{p}_{\mathrm{r} 1}^{\text {target }}-\mathrm{p}_{\mathrm{r} 2}^{\text {target }}\right)$

Where $p_{i}^{\text {trail }}$ is a trial vector generated for spider $i . p_{i}^{\text {target }}$ is the target vector associated with spider i. $\mathrm{p}_{\mathrm{r} 1}^{\text {target }}$ and $\mathrm{p}_{\mathrm{r} 2}^{\text {target }}$ are two target vectors associated with spiders $\mathrm{r} 1$ and $\mathrm{r} 2$, where $\mathrm{r} 1$ and $\mathrm{r} 2$ are two integer random numbers between 1 and $\mathrm{N}$, and $\mathrm{r} 1 \neq \mathrm{r} 2$. ri is a random number between zero and one. This number is drawn from a uniform distribution. $\otimes$ denotes element-wise multiplication. $\mathrm{Ki}$ is a binary vector for spider $\mathrm{i}$.

The elements of this binary vector are generated as follows:

$\mathrm{k}_{\mathrm{ij}}=\left\{\begin{array}{c}1 \text { if rand } \text { ij }_{\mathrm{j}}<\mathrm{C} \\ 0 \text { otherwise }\end{array}\right.$

Where $\mathrm{C}$ is a constant, $\mathrm{C} \in(0,1)$. rand $\mathrm{ij}_{\mathrm{j}}$ is the $\mathrm{j}$-th element of a random vector $\mathrm{i}$. This element is generated randomly between zero and one and obeys the uniform distribution. After generating 
the trail vector $i$, the position of spider $i$ in the next iteration is calculated using the following greedy selection process:

$\mathrm{p}_{\mathrm{i}}^{(\mathrm{t}+1)}=\left\{\begin{array}{c}\mathrm{p}_{\mathrm{i}}^{\text {trail }} \text { if } \mathrm{f}\left(\mathrm{p}_{\mathrm{i}}^{\text {trail }}\right)<\mathrm{f}\left(\mathrm{p}_{\mathrm{i}}\right) \\ \mathrm{p}_{\mathrm{i}} \text { otherwise }\end{array}\right.$

\section{Enhanced Spider Algorithm for reactive power problem}

1) Allocate values to the parameters

2) Generate the population of spiders pop and allocate memory for them.

3) Initialize vtar for every spider.

4) while stopping criteria not met do

5) For every spider $s$ in pop do

6) Calculate the fitness value of $s$.

7) Produce a vibration at the position of $s$.

8) end for

9) For every spider $\mathrm{s}$ in pop do

10) Compute the concentration of the vibrations (V) created by other spiders.

11) Choose the strongest vibration vbest from $V$.

12) if the concentration of vbest is larger than vtar then

13) Gather vbest as vtar.

14) end if

15) Carry out an arbitrary walk towards vtar.

16) Produce a arbitrary number $r$ from $[0,1]$.

17) if $\mathrm{r}<\mathrm{pj}$ then

18) Allot an arbitrary position to s.

19) end if

20) Alleviate the concentration of vtar.

21) end for

22) end while

23) Display Output - when best solution found.

\section{Simulation Results}

At first Enhanced Spider (ES) algorithm has been tested in standard IEEE-57 bus power system. The reactive power compensation buses are 18, 25 and 53. Bus 2, 3, 6, 8, 9 and 12 are PV buses and bus 1 is selected as slack-bus. The system variable limits are given in Table 1.

The preliminary conditions for the IEEE-57 bus power system are given as follows:

Pload = 12.182 p.u. Qload = 3.094 p.u.

The total initial generations and power losses are obtained as follows:

$\sum \mathrm{P}_{\mathrm{G}}=12.438$ p.u. $\sum \mathrm{Q}_{\mathrm{G}}=3.3152$ p.u.

Ploss $=0.25879$ p.u. Qloss $=-1.2081$ p.u.

Table 2 shows the various system control variables i.e. generator bus voltages, shunt capacitances and transformer tap settings obtained after optimization which are within the acceptable limits. In Table 3, shows the comparison of optimum results obtained from proposed methods with other 
optimization techniques. These results indicate the robustness of proposed approaches for providing better optimal solution in case of IEEE-57 bus system.

Table 1: Variable Limits

\begin{tabular}{|c|c|c|c|c|c|c|c|c|c|}
\hline \multicolumn{10}{|c|}{ Reactive Power Generation Limits } \\
\hline Bus no & 1 & \multicolumn{2}{|c|}{2} & 3 & 6 & 8 & \multicolumn{2}{|l|}{9} & 12 \\
\hline Qgmin & -1.4 & \multicolumn{2}{|c|}{-.015} & -.02 & -0.04 & -1.3 & \multicolumn{2}{|c|}{-0.03} & -0.4 \\
\hline Qgmax & 1 & \multicolumn{2}{|c|}{0.3} & 0.4 & 0.21 & 1 & \multicolumn{2}{|c|}{0.04} & 1.50 \\
\hline \multicolumn{10}{|c|}{ Voltage And Tap Setting Limits } \\
\hline vgmin & \multicolumn{2}{|c|}{ Vgmax } & \multicolumn{2}{|c|}{ vpqmin } & \multicolumn{2}{|c|}{ Vpqmax } & tkmin & \multicolumn{2}{|c|}{ tkmax } \\
\hline 0.9 & \multicolumn{2}{|c|}{1.0} & \multicolumn{2}{|c|}{0.91} & 1.05 & & & \multicolumn{2}{|c|}{1.0} \\
\hline \multicolumn{7}{|c|}{ Shunt Capacitor Limits } & .9 & & \\
\hline Bus no & \multicolumn{2}{|c|}{18} & \multicolumn{2}{|c|}{25} & \multicolumn{2}{|l|}{53} & & & \\
\hline Qcmin & \multicolumn{2}{|l|}{0} & \multicolumn{2}{|c|}{0} & \multicolumn{2}{|l|}{0} & & & \\
\hline Qcmax & \multicolumn{2}{|l|}{10} & \multicolumn{2}{|c|}{5.2} & \multicolumn{2}{|l|}{6.1} & & & \\
\hline
\end{tabular}

Table 2: Control variables obtained after optimization

\begin{tabular}{|l|l|}
\hline $\begin{array}{l}\text { Control } \\
\text { Variables }\end{array}$ & ES \\
\hline V1 & 1.1 \\
\hline V2 & 1.031 \\
\hline V3 & 1.034 \\
\hline V6 & 1.022 \\
\hline V8 & 1.021 \\
\hline V9 & 1.009 \\
\hline V12 & 1.016 \\
\hline Qc18 & 0.0668 \\
\hline Qc25 & 0.200 \\
\hline Qc53 & 0.0471 \\
\hline T4-18 & 1.009 \\
\hline T21-20 & 1.042 \\
\hline T24-25 & 0.865 \\
\hline T24-26 & 0.874 \\
\hline T7-29 & 1.050 \\
\hline T34-32 & 0.870 \\
\hline T11-41 & 1.018 \\
\hline T15-45 & 1.030 \\
\hline T14-46 & 0.910 \\
\hline T10-51 & 1.020 \\
\hline T13-49 & 1.060 \\
\hline T11-43 & 0.910 \\
\hline T40-56 & 0.900 \\
\hline T39-57 & 0.950 \\
\hline T9-55 & 0.950 \\
\hline
\end{tabular}


Table 3: Comparison results

\begin{tabular}{|l|l|l|l|l|}
\hline S.No. & $\begin{array}{l}\text { Optimization } \\
\text { Algorithm }\end{array}$ & Finest Solution & Poorest Solution & $\begin{array}{l}\text { Normal } \\
\text { Solution }\end{array}$ \\
\hline 1 & NLP [12] & 0.25902 & 0.30854 & 0.27858 \\
\hline 2 & CGA [12] & 0.25244 & 0.27507 & 0.26293 \\
\hline 3 & AGA [12] & 0.24564 & 0.26671 & 0.25127 \\
\hline 4 & PSO-w [12] & 0.24270 & 0.26152 & 0.24725 \\
\hline 5 & PSO-cf [12] & 0.24280 & 0.26032 & 0.24698 \\
\hline 6 & CLPSO [12] & 0.24515 & 0.24780 & 0.24673 \\
\hline 7 & SPSO-07 [12] & 0.24430 & 0.25457 & 0.24752 \\
\hline 8 & L-DE [12] & 0.27812 & 0.41909 & 0.33177 \\
\hline 9 & L-SACP-DE [12] & 0.27915 & 0.36978 & 0.31032 \\
\hline 10 & L-SaDE [12] & 0.24267 & 0.24391 & 0.24311 \\
\hline 11 & SOA [12] & 0.24265 & 0.24280 & 0.24270 \\
\hline 12 & LM [13] & 0.2484 & 0.2922 & 0.2641 \\
\hline 13 & MBEP1 [13] & 0.2474 & 0.2848 & 0.2643 \\
\hline 14 & MBEP2 [13] & 0.2482 & 0.283 & 0.2592 \\
\hline 15 & BES100 [13] & 0.2438 & 0.263 & 0.2541 \\
\hline 16 & BES200 [13] & 0.3417 & 0.2486 & 0.2443 \\
\hline 17 & Proposed ES & 0.22072 & 0.23016 & 0.22208 \\
\hline
\end{tabular}

Then Enhanced Spider (ES) algorithm has been tested in standard IEEE 118-bus test system [14]. The system has 54 generator buses, 64 load buses, 186 branches and 9 of them are with the tap setting transformers. The limits of voltage on generator buses are $0.95-1.1$ per-unit., and on load buses are $0.95-1.05$ per-unit. The limit of transformer rate is $0.9-1.1$, with the changes step of 0.025 . The limitations of reactive power source are listed in Table 4, with the change in step of 0.01 .

Table 4: Limitation of reactive power sources

\begin{tabular}{|l|l|l|l|l|l|l|l|}
\hline BUS & 5 & 34 & 37 & 44 & 45 & 46 & 48 \\
\hline QCMAX & 0 & 14 & 0 & 10 & 10 & 10 & 15 \\
\hline QCMIN & -40 & 0 & -25 & 0 & 0 & 0 & 0 \\
\hline BUS & 74 & 79 & 82 & 83 & 105 & 107 & 110 \\
\hline QCMAX & 12 & 20 & 20 & 10 & 20 & 6 & 6 \\
\hline QCMIN & 0 & 0 & 0 & 0 & 0 & 0 & 0 \\
\hline
\end{tabular}

The statistical comparison results of 50 trial runs have been list in Table 5 and the results clearly show the better performance of proposed Enhanced Spider (ES) algorithm in reducing the real power loss.

Table 5: Comparison results

\begin{tabular}{|l|l|l|l|l|}
\hline Active power loss (MW) & $\begin{array}{l}\text { BBO } \\
{[15]}\end{array}$ & $\begin{array}{l}\text { ILSBBO/ } \\
\text { strategy1 }\end{array}$ & $\begin{array}{l}\text { ILSBBO/ } \\
\text { strategy1 }\end{array}$ & $\begin{array}{l}\text { Proposed } \\
\text { ES }\end{array}$ \\
\hline
\end{tabular}




\begin{tabular}{|l|l|l|l|l|}
\hline & & {$[15]$} & {$[15]$} & \\
\hline Min & 128.77 & 126.98 & 124.78 & 117.04 \\
\hline Max & 132.64 & 137.34 & 132.39 & 120.01 \\
\hline Average & 130.21 & 130.37 & 129.22 & 118.62 \\
\hline
\end{tabular}

\section{Conclusion}

In this paper, one of the recently developed stochastic Enhanced Spider (ES) algorithm has been demonstrated and applied to solve reactive power problem. The proposed approach has been tested on the IEEE standard IEEE 57,118 bus systems and the simulation results indicate the effectiveness and robustness of the proposed Enhanced Spider (ES) algorithm in reducing the real power loss and particularly voltage profile are within the limits.

\section{References}

[1] M. A. Abido, J. M. Bakhashwain, "A novel multi objective evolutionary algorithm for optimal reactive power dispatch problem," in proc. Electronics, Circuits and Systems conf., vol. 3, pp. 1054-1057, 2003.

[2] W. N. W. Abdullah, H. Saibon, A. A. M. Zain, K. L. Lo, "Genetic Algorithm for Optimal Reactive Power Dispatch," in proc. Energy Management and Power Delivery conf., vol. 1, pp. 160-164, 1998.

[3] K. Y. Lee, Y. M. Park, J. L. Ortiz, "Fuel-cost minimisation for both real and reactive-power dispatches," in proc. Generation, Transmission and Distribution conf., vol. 131, pp. 85-93, 1984.

[4] S. Granville, "Optimal Reactive Dispatch Trough Interior Point Methods," IEEE Trans. on Power Systems, vol. 9, pp. 136-146, 1994.

[5] N. I. Deeb, S. M. Shahidehpour, "An Efficient Technique for Reactive Power Dispatch Using a Revised Linear Programming Approach,” Electric Power System Research, vol. 15, pp. 121-134, 1988.

[6] N. Grudinin, "Reactive Power Optimization Using Successive Quadratic Programming Method," IEEE Trans. on Power Systems, vol. 13, pp. 1219-1225, 1998.

[7] M. A. Abido, "Optimal Power Flow Using Particle Swarm Optimization," Electrical Power and Energy Systems, vol. 24, pp. 563-571, 2002.

[8] A. A. Abou El Ela, M. A. Abido, S. R. Spea, "Differential Evolution Algorithm for Optimal Reactive Power Dispatch,” Electric Power Systems Research, vol. 81, pp. 458-464, 2011.

[9] V. Miranda, N. Fonseca, "EPSO-Evolutionary Particle Swarm Optimization, A New Algorithm with Applications in Power Systems," in Proc. of Transmission and Distribution conf., vol. 2, pp. 745-750,2002.

[10] C.A. Canizares, A.C.Z.de Souza and V.H. Quintana, "Comparison of performance indices for detection of proximity to voltage collapse ," vol. 11. no.3 , pp.1441-1450, Aug 1996.

[11] C. F. Schaber, S. N. Gorb, and F. G. Barth, "Force transformation in spider strain sensors: White light interferometry.” J. Royal Society Interface, vol. 9, no. 71, pp. 1254-1264, Jun. 2012.

[12] Chaohua Dai, Weirong Chen, Yunfang Zhu, and Xuexia Zhang, "Seeker optimization algorithm for optimal reactive power dispatch," IEEE Trans. Power Systems, Vol. 24, No. 3, August 2009, pp. 1218-1231.

[13] J. R. Gomes and 0. R. Saavedra, "Optimal reactive power dispatch using evolutionary computation: Extended algorithms,” IEE Proc.-Gener. Transm. Distrib. Vol. 146, No. 6. Nov. 1999.

[14] IEEE, "The IEEE 30-bus test system and the IEEE 118-test system", (1993), http://www.ee.washington.edu/trsearch/pstca/. 
[15] Jiangtao Cao, Fuli Wang and Ping Li, "An Improved Biogeography-based Optimization Algorithm for Optimal Reactive Power Flow" International Journal of Control and Automation Vol.7, No.3 (2014), pp.161-176.

\footnotetext{
*Corresponding author.

E-mail address: gklenin@ gmail.com
} 